\title{
Formation of ice particles through nucleation in the mesosphere
}

\author{
Kyoko K. Tanaka ${ }^{1}$, Ingrid Mann ${ }^{2}$, and Yuki Kimura ${ }^{3}$ \\ ${ }^{1}$ Astronomical Institute, Tohoku University, 6-3, Aza Aoba, Aramaki, Aoba-ku, Sendai, 985-8578, Japan \\ ${ }^{2}$ Institute of Physics and Techonlogy, Department of Physics and Technology, University of Troms $\emptyset$ \\ ${ }^{3}$ Institute of Low Temperature Sciences, Hokkaido University, Kita-19, Nishi-8, Kita-ku, Sapporo, 060-0819, Japan \\ Correspondence: Kyoko K. Tanaka (kktanaka@astr.tohoku.ac.jp)
}

\begin{abstract}
.
Observations of polar mesospheric clouds have revealed the presence of solid ice particles in the upper mesosphere at high latitudes; however, their formation mechanism remains uncertain. In this study, we investigated the formation process of ice particles through nucleation from small amounts of water vapor at low temperatures. Previous studies that used classical nucle-

5 ation theory have shown that amorphous solid water particles can nucleate homogeneously at conditions that are present in the mesosphere. However, the rate predictions for water in classical nucleation theory disagree with experimental measurements by several orders of magnitude. We adopted a semi-phenomenological model for the nucleation process, which corrects the evaluation of the molecular cluster formation energy using the second virial coefficient, which agrees with both experiments and molecular dynamics simulations. To calculate the nucleation process, we applied atmospheric conditions for the temperature, pressure, numerical density of dust grains, and cooling rate. The results indicate that homogeneous water nucleation is extremely unlikely to occur in the mesosphere, while heterogeneous nucleation occurs effectively. Dust grains generated by meteor ablation can serve as nuclei for heterogeneous nucleation. We also showed that the ice can form directly in a crystalline state, rather than an amorphous state.
\end{abstract}

\section{Introduction}

The summer polar mesosphere, located at altitudes of 80-90 km, is the coldest part of the Earth's atmosphere. Clouds of ice particles can form at such heights, some of which are visible from the ground and are referred to as noctilucent clouds (Vaste, 1993). Noctilucent clouds are generally observed before sunrise and after sunset. Under similar conditions and at overlapping heights, strong radar echoes are observed, known as polar mesospheric summer echoes. Noctilucent clouds are related to the presence of water ice particles (Rapp and Lübken, 2004). Ice particles, also known as polar mesospheric clouds, have recently been observed by satellites (Hervig et al., 2012). The ice particles observed in noctilucent clouds comprise particles that are typically tens of nanometers in size (e.g., Gumbel and Merner, 2009), which are large enough to scatter light effectively and therefore, can be detected using a variety of optical remote sensing methods. Noctilucent clouds were considered to exist, however, they were difficult to observe visually before the twentieth century. The number of noctilucent clouds that are observed at high latitudes increases in the modern era over the years; it is suggested that this is because of the rise of $\mathrm{H}_{2} \mathrm{O}$ concentration and that noctilucent clouds are long-term indicators for climate change (e.g., Lübken et al., 2018). 
During summer, the high-altitude upper mesosphere can reach temperatures of $130 \mathrm{~K}$. Propagating gravity waves disturb the vertical temperature profiles within the mesospheric cloud layer (Dalin et al., 2012). The temperature at this altitude is highly variable and can reach a minimum of $100 \mathrm{~K}$ (Lübken, 1999; Rapp et al., 2002). At this low temperature, even a very small amount of water vapor can achieve a supersaturated state, indicating that water vapor can nucleate and particles can grow. The ice particles grow further as they sediment and are transported vertically in the atmosphere (Rapp et al., 2002), and can encounter different ambient temperatures. However, the exact ice particle formation process remains uncertain. Clouds in the troposphere are usually created by a heterogeneous nucleation, with aerosols as the nuclei. However, homogeneous nucleation in the mesosphere is thought to be possible because few impurities are present for heterogeneous nucleation (Zasetsky et al., 2009; Murray and Jensen, 2010). Thus, there are two possibilities for the ice particle formation. The first is heterogeneous nucleation, which requires sufficient nuclei, such as dust grains, on which the water vapor deposits. The second is homogeneous nucleation, wherein new water nuclei are formed directly from the gas phase if insufficient impurities are present.

Recent observational results support the hypothesis that ice particles in the mesosphere form as a result of heterogeneous nucleation. Satellite measurements of the atmosphere can be explained using ice particles that contain smaller particles, presumably meteoric smoke. Meteoric smoke particles consist of sodium bicarbonate, sodium hydroxide, soot, sulfuric acid, and proton hydrates, although there is insufficient evidence to determine which of these is dominant (Rapp and Thomas, 2006). Meteoric smoke particles form as a result of meteoroid ablation at altitudes of 70-110 km, and the resulting vapor condenses into agglomerates with radii of 0.1 to $2 \mathrm{~nm}$ (Hunten et al., 1980; Megner et al., 2006), which can subsequently be used for ice particle formation. The meteoric smoke provides deposition nuclei for ice particle formation. Hervig et al. (2012) considered the measured extinction of sunlight in the atmosphere due to the presence of ice particles that include fractions of meteoric smoke and found that the volume filling factor of meteoric smoke particles inside ice particles ranges from $0.05 \%$ to several percent. From in-situ rocket observations, Antonsen et al. (2017) determined the size distribution of meteoric smoke particles embedded in larger ice particles, which can be described by inverse power laws with exponents of 3.3-3.7. Experimental studies have also shown that heterogeneous nucleation is possible. Duft et al. (2019) measured heterogeneous ice deposition on iron silicate particles, which they considered to be analogous to meteoric smoke. The solid particles in the mesosphere are involved in atmospheric air circulation. During this process, coagulation growth can occur (Bardeen et al., 2008, 2010; Megner et al., 2008) and can also be influenced by interaction forces, which depend on the charge state (Baptiste et al., 2021). However, the deposition process remains the critical initial step, and its role in comparison with other growth processes remains uncertain.

Theoretical studies have shown that solid water particles can nucleate homogeneously at mesospheric conditions (Zasetsky et al., 2009; Murray and Jensen, 2010). Murray and Jensen (2010)suggested that the direct homogeneous nucleation of amorphous solid water (ASW) from the vapor phase is possible. They presented a parameterization of homogeneous nucleation based on a modified nucleation theory, wherein they adopted the classical nucleation theory from the vapor phase to ASW, although ASW is considered to be a meta-stable phase. They also showed that homogeneous nucleation competes with heterogeneous nucleation on meteoric smoke particles when the cooling rate is high $\left(>0.5 \mathrm{~K} \mathrm{~h}^{-1}\right)$. While the classical nucleation theory $(\mathrm{CNT})$ is the most widely used model for describing homogeneous nucleation, it is highly uncertain. It is known that rate 
predictions based on CNT disagree with experimental measurements for many substances. In the case of water, this deviation is a factor of 10-1000 (Dillmann and Meier, 1991).

A variety of theoretical approaches have been used to develop nucleation theory in previous studies. One of the most successful and useful models is the semi-phenomenological (SP) model, which corrects the formation energy evaluation of a cluster in CNT using the second virial coefficient of a vapor (Dillmann and Meier, 1991). The predictions obtained from the SP model agree surprisingly well with the experimental data for water, nonane, and n-alcohols. In the case of water, the experimental nucleation rate was one to three orders of magnitude smaller than that obtained using CNT, while the SP model was in good agreement within one order of magnitude (Dillmann and Meier, 1991).

In addition to laboratory experiments, numerical approaches, including molecular dynamics simulations, are a powerful method for testing the nucleation model, because the molecular kinetics can be analyzed in detail. To test nucleation theories, molecular dynamics simulations of water vapor nucleation have been performed. A comparison of nucleation models indicates that CNT overestimates nucleation rates by a few orders of magnitude, while the SP model exhibits a better performance (Tanaka et al., 2014; Angelil et al., 2015). Direct large molecular dynamics simulations of homogeneous water nucleation (using up to $4 \times 10^{6}$ molecules) have allowed extremely low and accurate nucleation to be measured (Angelil et al., 2015). A comparison with nucleation models also indicates the validity of the SP model. The results obtained by previous studies may change when a modified model is applied to the nucleation process in the atmosphere. Although many studies have addressed the validity of models of nucleation rates at fixed temperatures, few studies have investigated the changes that occur when these models are applied to natural phenomena where the temperature varies over time. Therefore, it is critical to investigate the effect of using a modified model on the nucleation process.

In this study, we reconsidered the homogeneous and heterogeneous nucleation mechanisms of ice particles in the mesosphere, with the aim of clarifying the formation mechanism of noctilucent clouds. In particular, we used a model for homogeneous nucleation that agrees with experimental and molecular dynamics simulations, and investigated the effects of using different models to clarify how the modified model affected previous results. We calculated a nucleation process in the cooling vapor using the SP model instead of CNT. The nucleation process depends on atmospheric conditions, including atmospheric temperature, pressure, and cooling rate. We described the homogeneous nucleation process of water droplets from water vapor based on the SP model and solved the temporal evolution of homogeneous nucleation throughout the cooling process. We also investigated the competition process between homogeneous and heterogeneous nucleation at various conditions. We investigated the heterogeneous nucleation process by comparing the parameters to the size distribution and amount of meteoric smoke particles reported by recent studies; however, this study does not consider their properties in detail. Therefore, we use the term "dust" in this study, as the results are generally applicable for solid particles. The conditions under which heterogeneous nucleation occurs effectively depend on the amount of dust grains and the cooling rate. Thus, we compared the derived conditions required for heterogeneous nucleation with previous observations. We also discuss the particle crystallization process using the crystallization timescale. 


\section{Methods}

\subsection{Homogeneous nucleation rate}

We first considered a formation process of ice particles due to homogeneous nucleation. When the partial pressure of the water vapor is larger than the equilibrium vapor pressure and becomes supersaturated, water molecules aggregate to form clusters. Cluster growth is promoted when the clusters reach and exceed a critical size. The nucleation rate, which is the number of generated critical clusters in a unit time and volume, is expressed in terms of the free energy of cluster formation (Kalikmanov, 2013). According to the nucleation theory, the nucleation rate $J$ is:

$100 J=\left[\sum_{i=1}^{\infty} \frac{1}{R^{+}(i) n_{e}(i)}\right]^{-1} \simeq R^{+}\left(i_{*}\right) n_{e}\left(i_{*}\right) Z$,

where $R^{+}(i)$ is the transition rate from a cluster of $i$ molecules, $i$-mer, to $(i+1)$-mer per unit time, i.e., the accretion rate, $n_{e}(i)$ is the equilibrium number density of $i$-mer, and $Z$ is the Zeldovich factor. $R^{+}(i)$ is given by $R^{+}(i)=\alpha n_{1} v_{\mathrm{th}}\left(4 \pi r_{0}^{2} i^{2 / 3}\right)$, where $\alpha$ is the sticking probability, $v_{\text {th }}$ is the thermal velocity $(=\sqrt{k T /(2 \pi m)}), n_{1}$ is the number density of the monomers. $r_{0}$ is the radius of a monomer $\left(=\left(3 m / 4 \pi \rho_{\mathrm{m}}\right)^{1 / 3}\right)$ where $m$ is the mass of a molecule and $\rho_{\mathrm{m}}$ is the bulk density. The equilibrium size distribution of a cluster is directly related to the free energy of cluster formation, $\Delta G_{i}$ :

$\frac{\Delta G_{i}}{k T}=\ln \left(\frac{n_{1}}{n_{\mathrm{e}}(i)}\right)$.

There exist three models for the formation energies $\Delta \mathrm{Gi}$, the classical nucleation theory (CNT), the modified classical nucleation theory (MCNT), and the semi-phenomenological (SP) model (Dillmann and Meier, 1991; Laaksonen et al., 1994). In each model, the free energy, $\Delta G_{i}$, is expressed as:

$110 \frac{\Delta G_{i}}{k T}=-i \ln S+\eta i^{2 / 3}$,

$\frac{\Delta G_{i}}{k T}=-(i-1) \ln S+\eta\left(i^{2 / 3}-1\right)$,

$\frac{\Delta G_{i}}{k T}=-(i-1) \ln S+\eta\left(i^{2 / 3}-1\right)+\xi\left(i^{1 / 3}-1\right)$,

where $S=P_{1} / P_{\text {sat }}$ is the supersaturation ratio of monomers using the saturated vapor pressure $P_{\text {sat }}$ and the partial pressure of monomers $P_{1} ; \eta$ and $\xi$ are temperature-dependent quantities that can be fixed from the condensed phase surface tension, bulk density and the second virial coefficient (Tanaka et al., 2014). Note that CNT assumes large cluster sizes, it is not expected to work for small clusters. In addition, its $\Delta G_{\mathrm{i}}$ does not vanish at $i=1$, i.e., for monomers, while MCNT and SP models satisfy $\Delta G_{i}=0$ at $i=1$. For the thermodynamic quantities, including the surface tension and the saturated vapor pressure of water, we used the data of amorphous ice (Murray and Jensen, 2010). $\eta$ is given by

$\eta=4 \pi r_{0}^{2} \gamma / k T$,

where $\gamma$ is the surface tension of the condensed phase. It has been suggested that when homogeneous nucleation occurs, the condensate is likely to be amorphous ice or supercooled droplets (Manka et al., 2012; Murray and Jensen, 2010), so the value 


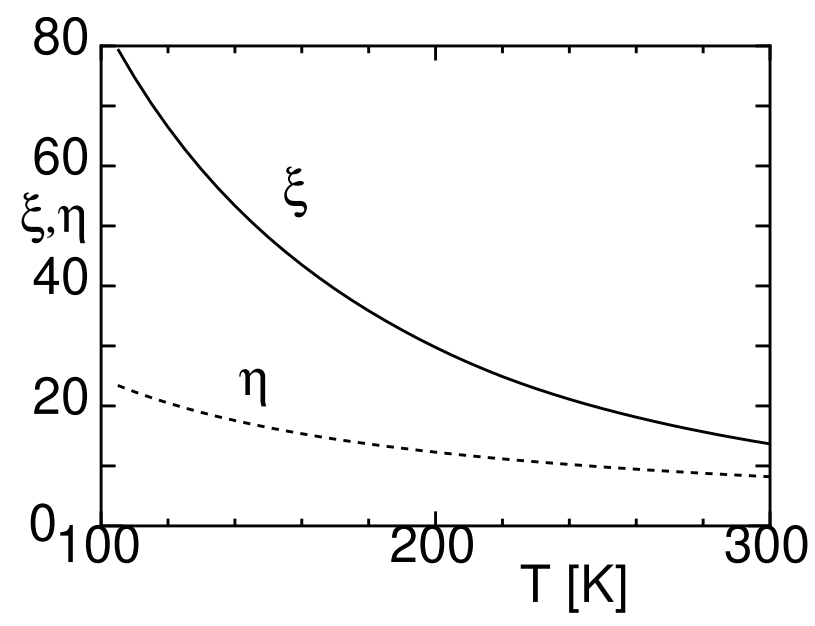

Figure 1. Dimensionless parameters $\eta$ and $\xi$ (used to calculate the nucleation rate) and their variations with temperature.

of amorphous ice (or supercooled droplet) is used in this study. As for the surface tension, we adopt the data of Murphy and Koop (2005) and Murray and Jensen (2010):

$$
\begin{aligned}
P_{\text {sat }}= & \exp [54.842763-6763.22 / T-4.21 \ln T+0.000367 T+ \\
& \tanh \{0.415(T-218.8)\}(53.878-1331.22 / T-9.44523+0.014025 T)][\mathrm{Pa}] \\
\gamma= & 235.8\left(\frac{T_{\mathrm{c}}-T}{T_{\mathrm{c}}}\right)^{1.256}\left[1-0.625\left(\frac{T_{\mathrm{c}}-T}{T_{\mathrm{c}}}\right)\right]\left[\operatorname{erg~cm}^{-2}\right],
\end{aligned}
$$

where the critical temperature of water $T_{\mathrm{c}}=647.15 \mathrm{~K}$. At $100-170 \mathrm{~K}$, which is the temperature range in this study, the difference in the surface tension is small $\left(87-90 \mathrm{erg}^{-2}\right)$.

The monomer radius is derived from the material density. We set $\rho_{\mathrm{m}}=0.93 \mathrm{gcm}^{-3}$ (Murray and Jensen, 2010). $\xi$ is a nondimensional parameter that depends on $T$, which was fixed using the second virial coefficient $B_{2}$. We fixed the parameter $\xi$ as

$$
\xi=-\frac{1}{2^{\frac{1}{3}}-1}\left[\ln \left(\frac{-B_{2} P_{\text {sat }}}{k T}\right)+\left(2^{\frac{2}{3}}-1\right) \eta\right] .
$$

and the second virial coefficient $\mathrm{B}_{2}\left[\mathrm{~cm}^{3} / \mathrm{mol}\right]$ is defined as:

$$
B_{2}=1000\left(0.34404 T_{*}^{-0.5}-0.758264 T_{*}^{-0.8}-24.219 T_{*}^{-3.35}-3978.2 T_{*}^{-8.3}\right),
$$

where $T_{*}=T / 100$ (Harvey and Lemmon, 2004). As shown in Fig. 1, both $\eta$ and $\xi$ increase as the temperature decreases. This indicates that the energy barrier for cluster formation increases because of the increase in $\Delta G_{i}$. In this case, the nucleation rate decreases. At $100-150 \mathrm{~K}$, the value of $\eta$ is approximately 10 , and the value of $\xi$ is approximately 50 . This indicates that nucleation occurrence is even more difficult than previously thought.

Using the nucleation rate described above, we solved the basic equations governing non-equilibrium condensation, wherein we considered a gaseous system that cools on a characteristic time scale $\tau$ (Yamamoto and Hasegawa, 1977; Tanaka et al., 
2002). The cooling time is defined as $\tau^{-1}=\left(-1 / T_{0}\right)(d T / d t)$, where $t$ and $T_{0}$ are the time and initial temperature, respectively. The basic equation describing ice particle growth is given as:

$\frac{\partial r\left(t, t^{\prime}\right)}{\partial t}=\alpha_{\mathrm{s}} n_{1}(t) v_{\mathrm{th}} \Omega_{1}$

where $r\left(t, t^{\prime}\right)$ is the radius formed by homogeneous nucleation at $t$ nucleated at time $t^{\prime}, v_{\text {th }}$ is the thermal velocity of the monomer, and $\Omega_{1}$ is the monomer volume. The equation describing the consumption of the monomers is as follows:

$n_{1}(t)=n_{1}(0)-\int_{0}^{t} J\left(t^{\prime}\right)\left(\frac{r\left(t, t^{\prime}\right)}{r_{1}}\right)^{3} d t^{\prime}$.

\subsection{Competing process between homogeneous and heterogeneous nucleations}

If sufficient dust grains are present in the cloud region, most water molecules will deposit on the surfaces of the dust grains. However, if the number of dust grains is insufficient, new nuclei form, i.e., homogeneous nucleation occurs. We evaluated the competing process between homogeneous and heterogeneous nucleation and obtained the conditions required for the occurrence of heterogeneous nucleation based on a simple analysis.

In particular, deposition depends on the interfacial energy between the vapor and dust substances. However, meteoric smoke particles are composed of metals and silicates (Rapp and Thomas, 2006; Plane et al., 2015) and water molecules are thought to deposit quickly on their surfaces (Duft et al., 2019). Therefore, we considered the interfacial energy to be sufficiently small to be negligible. We also assumed that the radii of the dust grains were larger than the critical cluster radius required for homogeneous nucleation, as the vapor will not deposit on the dust grains if their radii are smaller than the critical cluster radius, owing to the effect of the surface energy of water. As indicated above, the radius of the critical cluster is very small $\left(i_{*}=2-10\right)$, making this assumption reasonable.

Instead of Eq.(12), we used the equation describing the consumption of monomers, given as:

$$
\begin{aligned}
n_{1}(t)= & n_{1}(0)-\int_{0}^{t} J\left(t^{\prime}\right)\left(\frac{r\left(t, t^{\prime}\right)}{r_{1}}\right)^{3} d t^{\prime} \\
& -\int_{a_{\min }}^{a_{\max }} A a^{-\lambda}\left(\frac{r_{\mathrm{h}}^{3}-a^{3}}{r_{1}^{3}}\right) d a
\end{aligned}
$$

where $r_{\mathrm{h}}$ is the radius of a heterogeneous particle. We considered the dust grain size distribution $n_{\mathrm{d}}(a)$, given by $n_{\mathrm{d}}(a)=A a^{-\lambda}$, with a dust grain radius $a$ and an inverse power exponent $\lambda$, which was set to 2.5 or 3.5 based on observations in this study. The third term on the right-hand side of Eq. (13) corresponds to the monomer consumption, owing to the accretion of the monomer onto the dust grains, and $a_{\min }$ (or $a_{\max }$ ) is the minimum (maximum) radius of the dust grains, and the density of the dust grains is given by:

$$
\rho_{d}=\int_{a_{\min }}^{a_{\max }} \frac{4 \pi}{3} \rho_{\mathrm{m}} a^{3} n_{\mathrm{d}}(a) d a,
$$


where the constant $A$ in the size distribution is given by

$$
\begin{gathered}
A=\frac{3 \rho_{d}}{8 \pi \rho_{m} \sqrt{a_{\max }}-\sqrt{a_{\min }}} \text { for } \lambda=3.5, \text { and } \\
A=\frac{9 \rho_{d}}{8 \pi \rho_{m}\left(a_{\max }^{1.5}-a_{\min }^{1.5}\right)} \text { for } \lambda=2.5 .
\end{gathered}
$$

We considered the equations describing heterogeneous particle growth, i.e., particle consisting of a dust center and an outer layer of ice, as well as the homogeneous particles given by Eq. (11):

$\frac{d r_{\mathrm{h}}(t)}{d t}=\alpha_{\mathrm{s}} n_{1} v_{\mathrm{th}} \Omega_{1}$

where the initial radius of the heterogeneous particle corresponds to the radius of dust grains $r_{\mathrm{h}}(0)=a$.

We can determine whether homogeneous or heterogeneous nucleation is the dominant process based on the fraction of water molecules incorporated into the particle. We considered how much of the water molecule was consumed by heterogeneous nucleation before $t_{j}$, which is the time of the peak nucleation rate due to homogeneous nucleation. If the majority of the molecules have been consumed by heterogeneous nucleation at this time, heterogeneous nucleation is considered to be dominant. Therefore, we obtained the conditions at which the heterogeneous particle formation becomes effective:

$f=\frac{1}{n_{1}(0)} \int_{a_{\min }}^{a_{\max }} A r^{-\lambda}\left(\frac{r_{\mathrm{h}}\left(t_{j}\right)^{3}-a^{3}}{r_{1}^{3}}\right) d a \gtrsim 0.5$,

where $f$ is the fraction of water molecules consumed by the heterogeneous particles at $t_{j}$. Assuming that the number density of water molecules at $t_{j}$ is nearly equal to the initial value $n_{1}\left(t_{j}\right) \simeq n_{1}(0)$, the radius of a heterogeneous grain is:

$$
\begin{aligned}
r_{\mathrm{h}}(t) & \simeq a+\alpha_{\mathrm{s}} n_{1}(0) v_{\mathrm{th}} \Omega_{1} t_{j} \\
& =a+\frac{r_{1}}{3} \frac{t_{j}}{\tau_{\mathrm{col}}} .
\end{aligned}
$$

Inserting the above equation into Eq. (18), we obtain:

$f=\frac{A}{n_{1}(0)} \int_{a_{\min }}^{a_{\max }} a^{-\lambda}\left[\left(\frac{a}{r_{1}}+\frac{t_{j}}{3 \tau_{\mathrm{col}}}\right)^{3}-\left(\frac{a}{r_{1}}\right)^{3}\right] d a \gtrsim 0.5$,

where $\tau_{\text {col }}=\left(4 \pi r_{1}^{2} \alpha_{\mathrm{s}} n_{\mathrm{v}}(0) v_{\text {th }}\right)^{-1}$ is the collision time among monomers. From Eq.(20), we obtain the following condition for $\lambda=2.5$.

$$
\begin{aligned}
\frac{A}{n_{1}(0)}\left[\frac { 6 } { r _ { 1 } ^ { 2 } } ( \frac { t _ { j } } { 3 \tau _ { \mathrm { col } } } ) \left(r_{\max }^{0.5}\right.\right. & \left.-r_{\min }^{0.5}\right)+\frac{6}{r_{1}}\left(\frac{t_{j}}{3 \tau_{\mathrm{col}}}\right)^{2}\left(r_{\min }^{-0.5}-r_{\max }^{-0.5}\right) \\
+ & \left.\frac{3}{2}\left(\frac{t_{j}}{3 \tau_{\mathrm{col}}}\right)^{3}\left(r_{\min }^{-1.5}-r_{\max }^{-1.5}\right)\right] \gtrsim 0.5
\end{aligned}
$$


which yields the conditions for the dust grain density when $\lambda=2.5 \lambda=2.5$ :

$$
\begin{aligned}
\rho_{\mathrm{d}} \gtrsim & 4 \pi \rho_{\mathrm{m}} n_{1}(0) C, \\
C= & \frac{\left(a_{\max }^{1.5}-a_{\min }^{1.5}\right)}{9}\left[\frac{6}{r_{1}^{2}}\left(\frac{t_{j}}{3 \tau_{\mathrm{col}}}\right)\left(a_{\max }^{0.5}-a_{\min }^{0.5}\right)+\frac{6}{r_{1}}\left(\frac{t_{j}}{3 \tau_{\mathrm{col}}}\right)^{2}\left(a_{\min }^{-0.5}-a_{\max }^{-0.5}\right)\right. \\
& \left.+\frac{3}{2}\left(\frac{t_{j}}{3 \tau_{\mathrm{col}}}\right)^{3}\left(a_{\min }^{-1.5}-a_{\max }^{-1.5}\right)\right]^{-1} \quad \text { for } \lambda=2.5 .
\end{aligned}
$$

In the same way, we obtained the condition when $\lambda=3.5$ :

$$
\begin{aligned}
C= & \frac{\left(a_{\max }^{0.5}-a_{\min }^{0.5}\right)}{3}\left[\frac{6}{r_{1}^{2}}\left(\frac{t_{j}}{3 \tau_{\mathrm{col}}}\right)\left(a_{\min }^{-0.5}-a_{\max }^{-0.5}\right)+\frac{2}{r_{1}}\left(\frac{t_{j}}{3 \tau_{\mathrm{col}}}\right)^{2}\left(a_{\min }^{-1.5}-a_{\max }^{-1.5}\right)\right. \\
& \left.+\frac{2}{5}\left(\frac{t_{j}}{3 \tau_{\mathrm{col}}}\right)^{3}\left(a_{\min }^{-2.5}-a_{\max }^{-2.5}\right)\right]^{-1} \quad \text { for } \lambda=3.5 .
\end{aligned}
$$

\section{Results}

\subsection{Homogeneous nucleation}

Figure 2 shows a typical example of the homogeneous nucleation at an initial temperature of $135 \mathrm{~K}$, where we solved the basic equations of Eqs. (11) and (12) using the nucleation rate given by Eq. (1). When the initial temperature is $135 \mathrm{~K}$, where the saturation vapor pressure and the number density of water molecules are $2.0 \times 10^{-7} \mathrm{~Pa}$ and $1.0 \times 10^{8} \mathrm{~cm}^{-3}$, respectively. In this case, considering an atmospheric pressure of $0.2-0.5 \mathrm{~Pa}$ at approximately $85 \mathrm{~km}$, the water vapor fraction corresponds to $0.4-1 \mathrm{ppmv}$. Similarly, if the temperature is $145 \mathrm{~K}$, the water vapor fraction corresponds to $5-20 \mathrm{ppmv}$. The observations indicate that there are some variations in the water content, and that the water vapor fraction in the atmosphere is $1-10 \mathrm{ppmv}$ (Berger and vonZahn, 2002; Lübken et al., 2004). Therefore, we considered $135 \mathrm{~K}$ and $145 \mathrm{~K}$ as typical values in this study. Figure 2 shows the behavior of non-equilibrium condensation of water with a characteristic cooling time of $\tau=1.5 \times 10^{5} \mathrm{~s}$, which corresponds to a cooling rate of $1.0 \times 10^{-3} \mathrm{Ks}^{-1}$, in which we used the SP model and assumed the sticking probability of a water molecule to be unity. Because the supersaturation ratio increases exponentially with a decrease in temperature, and because the nucleation rate depends strongly on the supersaturation ratio, the nucleation rate increased sharply. A slight decrease in water molecules due to nucleation caused the nucleation rate to reach its maximum $\left(1 \mathrm{~cm}^{-3} \mathrm{~s}^{-1}\right)$ at a temperature $T=T_{p}$. We call this peak temperature as the nucleation temperature, hereafter. The nucleation temperature $T_{p}$ was $63 \mathrm{~K}$, and the average radius of the water particles was $4.6 \mathrm{~nm}$. After nucleation, the nucleus grew rapidly, doubling the average radius in $\sim 7 \mathrm{~h}$.

215 The nucleation temperature and particle size depend on the nucleation model used for calculation. Figure 3 shows the temporal evolution of nucleation rates for the MCNT and SP models. The nucleation temperature was $63 \mathrm{~K}$ for the SP model, which was much smaller than the $106 \mathrm{~K}$ obtained when using the MCNT model. The average water droplet radii were 4.6 and $1.3 \mathrm{~nm}$ for the SP and MCNT models, respectively. A lower nucleation temperature was obtained for the SP model because the 


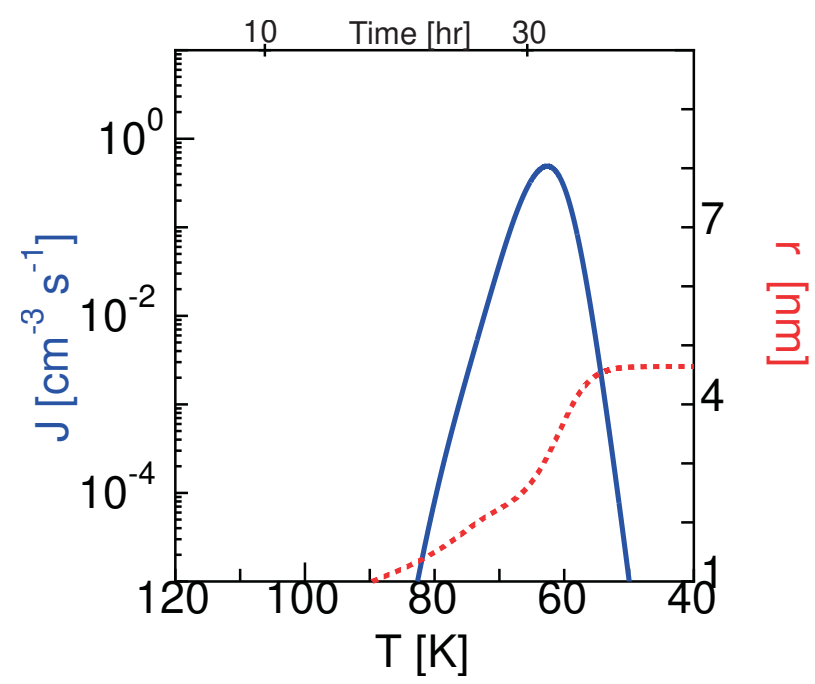

Figure 2. Time evolution of the nucleation rate and mean particle radius during homogeneous nucleation, calculated using the SP model. The initial temperature was $135 \mathrm{~K}$ and the cooling rate is $1 \times 10^{-3} \mathrm{Ks}^{-1}$.

free energy for cluster formation $\Delta G_{i}$ is much larger for the SP model than the MCNT model. Due to the high supersaturation ratio, the sizes of the critical clusters are very small in both models, i.e., two and four molecules for the SP and MCNT models, respectively.

When we performed the calculation using CNT, the nucleation temperature obtained was between those of the MCNT and SP models. For example, the nucleation temperature was $87 \mathrm{~K}$ when using CNT, which is between those of the MCNT (106 K) and SP $(63 \mathrm{~K})$ models. As noted above, CNT cannot accurately describe $\Delta G_{i}$ for monomers and has been corrected to be consistent in previous homogeneous nucleation studies. Therefore, we use MCNT instead of CNT for comparison with SP model in this study.

Figure 4 shows the results of the calculations at various cooling rates when the initial temperature was $135 \mathrm{~K}$. For MCNT, the nucleation temperature ranged from $\sim 100$ to $110 \mathrm{~K}$ at a cooling rate of $10^{-5} \mathrm{Ks}^{-1}$ to $10^{-2} \mathrm{Ks}^{-1}$; however, for the SP model, the nucleation temperature was as low as $100 \mathrm{~K}$. When the cooling rate was $10^{-5} \mathrm{Ks}^{-1}$, the nucleation temperature was $\sim 80$ $\mathrm{K}$. As the cooling rate increased, the nucleation temperature decreased, reaching $50 \mathrm{~K}$ for a cooling rate of $\sim 10^{-2} \mathrm{Ks}^{-1}$. Low nucleation temperatures do not match the observations, indicating that homogeneous nucleation is difficult in the mesosphere. In contrast, the size did not change drastically between the two models. For the SP model, the size was larger by a factor of 2. Figure 5 shows the results for an initial temperature of $145 \mathrm{~K}$. For MCNT, the nucleation temperature ranged from $\sim 110$ to $120 \mathrm{~K}$, but for the SP model, it was also as low as $100 \mathrm{~K}$. When the cooling rate was slow $\left(10^{-5} \mathrm{Ks}^{-1}\right)$, the nucleation temperature was $\sim 100 \mathrm{~K}$ in the SP model. For initial temperatures of $135 \mathrm{~K}$ and $145 \mathrm{~K}$, the initial amounts of water vapor were quite different; the amount of water vapor present was 20 times higher at $145 \mathrm{~K}$ than at $135 \mathrm{~K}$. However, the nucleation temperatures were lower for both cases. In contrast, the nucleation temperature was considered to be higher than $100 \mathrm{~K}$ based 


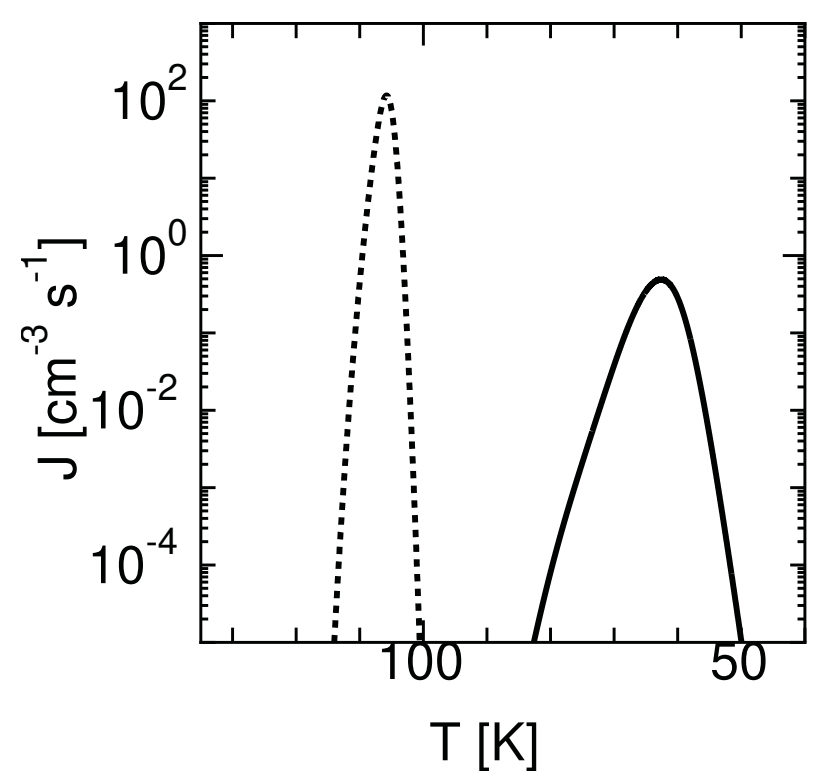

Figure 3. Time evolution of the nucleation rates of two models using homogeneous nucleation. The solid curve shows the results of the SP model, while the dotted curve shows the results of the MCNT model.

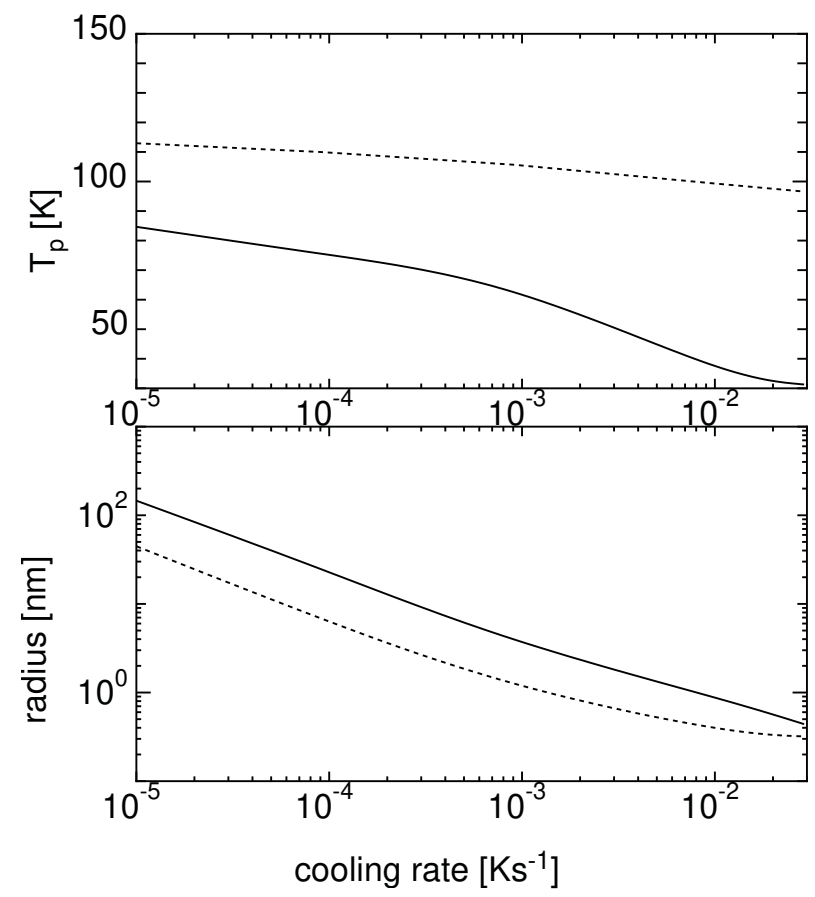

Figure 4. Nucleation temperatures and mean particle radii for homogeneous nucleation with an initial temperature of $135 \mathrm{~K}$. Solid curve shows the results of the SP model, dotted curve shows the results of the MCNT model. 


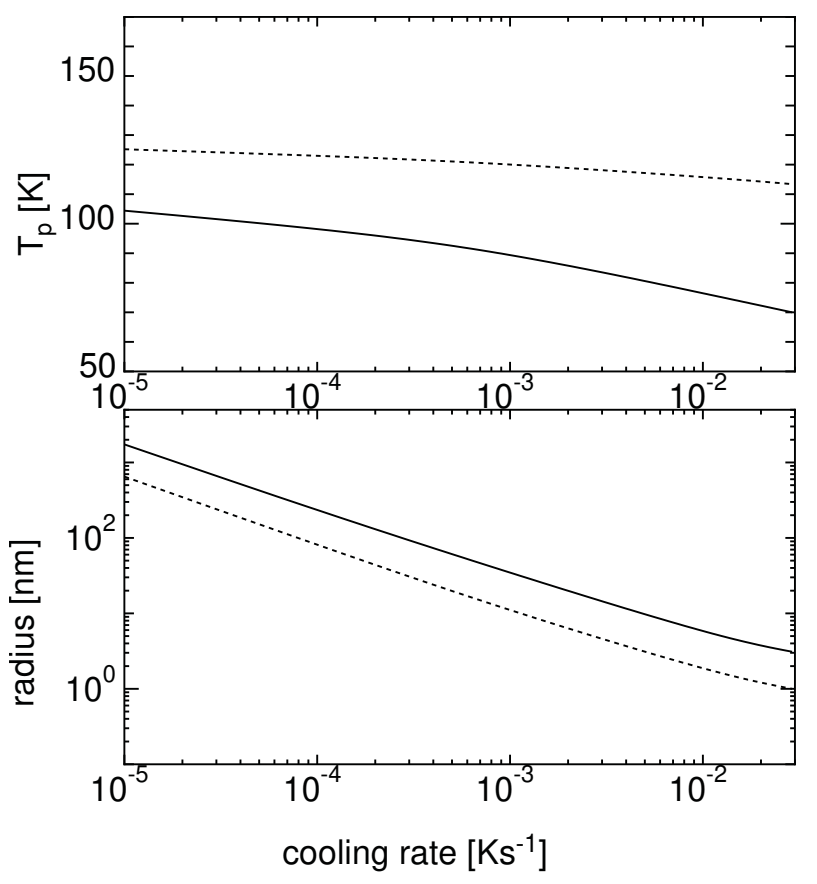

Figure 5. The same as Figure 4 but for an initial temperature of $145 \mathrm{~K}$.

on the observations. To nucleate water homogeneously at a reasonable temperature above $100 \mathrm{~K}$, the cooling rate must be slower than $10^{-6} \mathrm{Ks}^{-1}$ and $10^{-5} \mathrm{Ks}^{-1}$ for an initial temperature of $135 \mathrm{~K}$ or $145 \mathrm{~K}$.

\subsection{Condition for heterogeneous nucleation}

We investigated the competing process between homogeneous and heterogeneous nucleation and obtained the condition required for the occurrence of heterogeneous nucleation. Figures 6 and 7 show the dust grain density required for heterogeneous nucleation as a function of cooling time given by Eq. (22) when the initial temperatures are $135 \mathrm{~K}$ and $145 \mathrm{~K}$ and the time at which the homogeneous nucleation rate attains its peak is given by $t_{j} \simeq \tau$ (Yamamoto and Hasegawa, 1977). In Figs 6 and 7, we adopted $a_{\min }=0.2 \mathrm{~nm}$ and $a_{\max }=4 \mathrm{~nm}$ (Baumann et al., 2015). When the amount of dust is large, heterogeneous nucleation occurs. However, when cooling occurs rapidly, homogeneous nucleation is more effective because the supercooling ratio increases quickly. Figures 6 and 7 show a region where heterogeneous nucleation is dominant (where the cooling rate is smaller and the dust amount is larger), as well as a possible range in the mesospheric environment where a wide range of cooling rates occur $\left(10^{-2} \mathrm{Ks}^{-1}\left(\sim \mathrm{K} \mathrm{day}^{-1}\right)\right.$ to $\left.10^{-2} \mathrm{Ks}^{-1}\left(\sim \mathrm{K} \mathrm{min}{ }^{-1}\right)\right)$. In, Figures 6 and 7 , the range of density of the dust grains obtained from the observation is shown (Hervig et al., 2012; Antonsen et al., 2017). From Figures 6 and 7, it is clear that most of the observed region is dominated by heterogeneous nucleation, thereby indicating that heterogeneous nucleation is dominant in the mesosphere. 


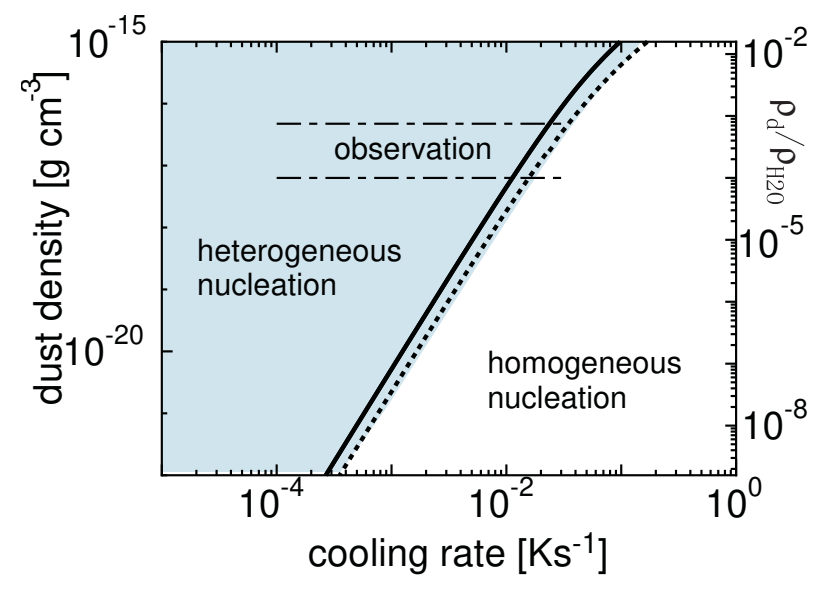

Figure 6. The condition of the amount of dust (vertical axis) and the cooling rate (horizontal axis) required for the heterogeneous nucleation at an initial temperature of $135 \mathrm{~K}$. Solid and dotted lines represent $\lambda=2.5$ and $\lambda=3.5$, respectively.

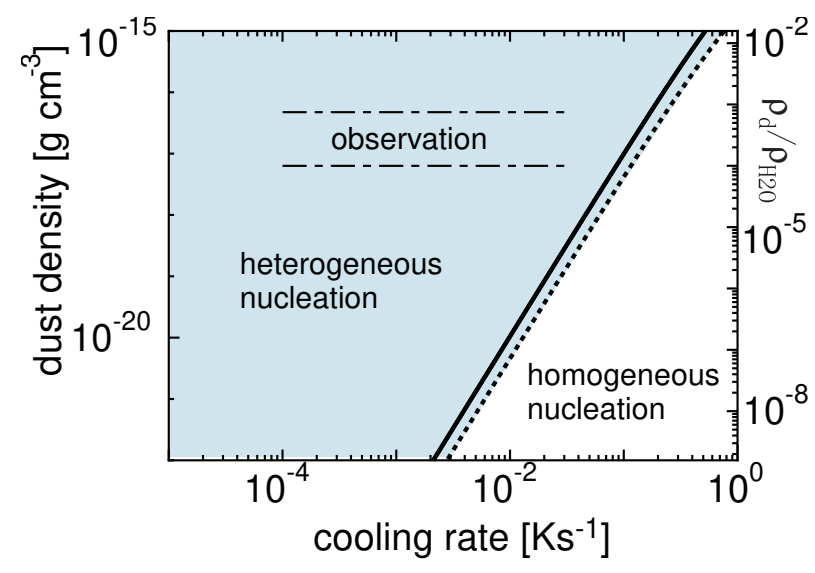

Figure 7. The same as Fig.6 but for an initial temperature of $145 \mathrm{~K}$.

\subsection{Crystallization process}

Ice exhibits two potential states when it nucleates in the mesosphere: amorphous or crystalline. However, the state of the ice remains unclear. When water nucleates homogeneously, the first transition is to an amorphous phase with an energetically lower barrier, rather than a stable phase, as described in the Ostwald step rule (Ostwald, 1879). However, experiments on the homogeneous nucleation of water at very low temperatures $(\sim 100 \mathrm{~K})$ have indicated that liquid water or amorphous ice forms (Manka et al., 2012). In contrast, during heterogeneous nucleation, the solid state depends on certain quantities, including pressure and temperature. We introduced a condition for amorphous ice formation based on a simple analysis. This condition was derived by previous studies (Gail and Sedlmayr, 1984; Kouchi et al., 1994), i.e., the diffusion distance of the coverage time of the surface by adatoms is smaller than the lattice constant $a_{1}\left(=4.5 \times 10^{-8} \mathrm{~cm}\right)$ of crystalline ice, which yields the following 


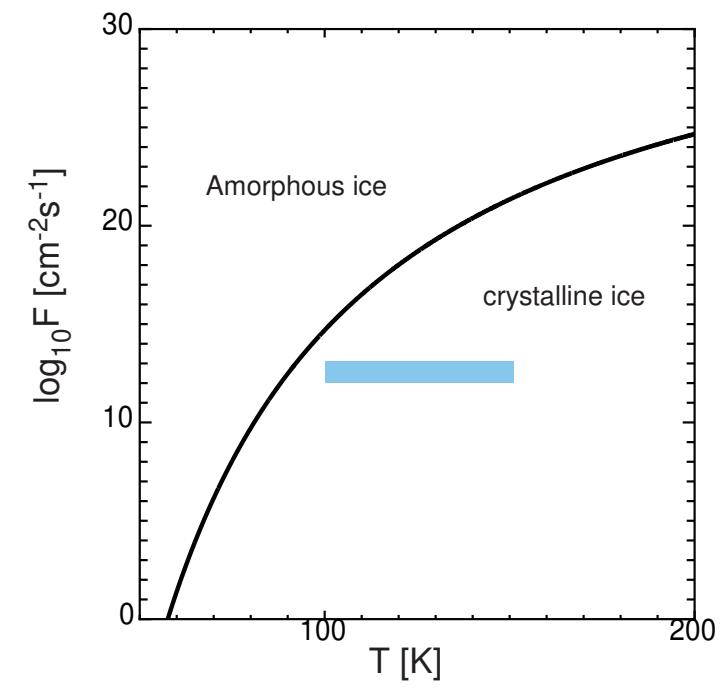

Figure 8. Flux of water molecules given by $F_{\mathrm{c}}$ and shown by the solid line. In the region larger than $F_{\mathrm{c}}$, the solid ice is considered to be amorphous, while in the smaller region it is crystalline. In the mesosphere, the flux of water molecules $F$ is approximately $10^{12}-$ $10^{13} \mathrm{~cm}^{-2} \mathrm{~s}^{-1}$ for the temperature range of $100 \mathrm{~K}-150 \mathrm{~K}$ (shaded region).

condition:

$F>D_{\mathrm{s}} / a_{1}^{4}=F_{\mathrm{c}}$

where $F$ is the flux of water molecules and $D_{\mathrm{s}}$ is the surface diffusion coefficient of the water molecules, which is given by $D_{\mathrm{s}}=D_{\mathrm{s} 0} \exp \left(-E_{\mathrm{s}} / k T\right)$. Figure 8 shows $F_{\mathrm{c}}$ in Eq. (25) as a function of temperature, where $E_{\mathrm{s}} / k=4590 \mathrm{~K}$ and $D_{\mathrm{s} 0}=$ $1.74 \times 10^{5} \mathrm{~cm}^{-2} \mathrm{~s}^{-1}$ (Kouchi et al., 1994). In the region larger than $F_{\mathrm{c}}$, the solid ice is considered to be amorphous. However, in the smaller region, the ice is crystalline. Figure 8 also shows the flux of water molecules on the dust surfaces $F$, which is assumed to be $F=n_{1} v_{\mathrm{th}}$. In the mesosphere, the flux of water molecules is $\sim 10^{12}-10^{13} \mathrm{~cm}^{-2} \mathrm{~s}^{-1}$ (shaded region in Fig. 8). This flux range corresponds to crystalline ice formation. The results indicate that the ice particles solidify as crystals when they nucleate at mesospheric conditions.

\section{Discussion and conclusion}

To explain the formation of clouds in the mesosphere, there are two possibilities: homogeneous and heterogeneous nucleations. We tested these two mechanisms theoretically. For homogeneous nucleation, we used the SP model, which agreed with the experiments and molecular dynamics simulations. The different nucleation models produce large differences in the nucleation process, mainly regarding the nucleation temperature. Using the nucleation rate obtained from the SP model, we calculated the time evolution of the number of water molecules and ice particle growth. Compared to the CNT model, the nucleation 
temperature was very low. At an initial temperature of $135 \mathrm{~K}$, the ice nucleation temperature was very low, ranging from 50 to $80 \mathrm{~K}$ (Fig. 4). When the initial temperature was $145 \mathrm{~K}$, the number density of water molecules and the nucleation temperature both increased, but the nucleation temperature was still below (Fig. 5). The nucleation temperature for homogeneous nucleation is far below $100 \mathrm{~K}$, and therefore, below typically observed temperatures. If the cooling rate was slower than $10^{-5} \mathrm{Ks}^{-1}$, then the nucleation temperature was above $100 \mathrm{~K}$. However, the cooling time in the mesosphere is a few days at most; thus, the cooling rate will not be that slow. Therefore, the potential for homogeneous nucleation in the mesosphere is considered to be very small, although previous studies have suggested that homogeneous nucleation can occur.

We also determined the conditions at which heterogeneous nucleation occurs and compared them with observational data. Our results indicate that heterogeneous nucleation occurs effectively in the mesosphere. Because dust from micrometeorites is present at this altitude, heterogeneous nucleation using fragments of micrometeorites as nuclei is considered to be the dominant process. As shown in Section 4, heterogeneous nucleation prevails even for a wide range of cooling rates and amounts of water in the mesosphere. When ice deposits due to heterogeneous nucleation, the growth rate is $(0.3-7) \times 10^{-3} \mathrm{nms}^{-1}$ from Eq. (17). This indicates that the radii of the particles increase to $1-25 \mathrm{~nm}$ in one hour. Since the clouds are observed on a timescale of a few hours, this rate is consistent with the observations. The particle growth rate becomes faster as the number density of water molecules increases; therefore, if rapid growth is observed, the number density of water molecules may need to be larger.

Our theoretical study shows that the formation of ice particles in the mesosphere by homogeneous nucleation is extremely difficult. It is therefore unlikely to be the major process for the formation of mesospheric cloud and noctilucent cloud particles. While homogeneous nucleation is unlikely to occur on Earth, the ice formation in the mesosphere is thought to be the most likely place on Earth for homogeneous nucleation to occur. Our results, however, suggest that there is no particle formation via homogeneous nucleation on Earth. On the other hand, the probability for heterogeneous nucleation is very high even for small fraction of dust being present. Our study also shows that during the deposition process, the ice can form directly in crystalline state rather than amorphous state. After nucleation, the coagulation process for the formation of larger ice particles, which needs to be investigated based on different theories, should be studied in future research. YK contributed to the discussion of the paper. All authors contributed to writing and editing the manuscript.

Competing interests. The authors declare no conflicts of interest associated with this manuscript.

Acknowledgements. This work was supported in part by JSPS KAKENHI Grants No. 20H05657, 19K03941, and 18K03689 and by Research Council of Norway Grant No. 275503. 


\section{References}

Angelil, R., Diemand, J., and Tanaka, K. K.and Tanaka, H.: Homogeneous SPC/E water nucleation in large molecular dynamics simulations, J. Chem. Phys., 143, 064 507, https://doi.org/10.1063/1.4928055, 2015.

Antonsen, T., Havnes, O., and Mann, I.: Estimates of the Size Distribution of Meteoric Smoke Particles From Rocket-Borne Impact Probes, Journal of Geophysical Research: Atmospheres, 122, 12,353-12,356, https://doi.org/10.1002/2017JD027220, 2017.

Baptiste, J., Williamson, C., Fox, J., Stace, A. J., Hassan, M., Braun, S., Stamm, B., Mann, I., and Besley, E.: The influence of surface charge on the coalescence of ice and dust particles in the mesosphere and lower thermosphere, Atmos. Chem. Phys., 21, 8735-8745,, https://doi.org/10.5194/acp-21-8735-2021, 2021.

Bardeen, C. G.and Toon, O. B., Jensen, E. J., Marsh, D. R., and Harvey, V. L.: Numerical simulations of the three-dimensional distribution of meteoric dust in the mesosphere and upper stratosphere, J. Geophy. Res., 113, D17 202, https://doi.org/10.1029/2007JD009515, 2008.

Bardeen, C. G.and Toon, O. B., Jensen, Harvig, M. E., Randall, C. E., S., B., Marsh, D. R., and Merkel, A.: Numerical simulations of the three-dimensional distribution of polar mesospheric clouds and comparisons with Cloud Imaging and Particle Size (CIPS) experiment and the Solar Occultation For Ice Experiment (SOFIE) observations, J. Geophy. Res., 115, D10 204, https://doi.org/10.1029/2009JD012451, 2010 .

Baumann, C., Rapp, M., Anttila, M., Kero, A., and Verronen, P. T.: Effects of meteoric smoke particles on the D region ion chemistry, J. Geophy. Res. Space Physics, 120, 10,823-10,839, https://doi.org/10.1002/2015JA021927, 2015.

Berger, U. and vonZahn, U.: Icy particles in the summer mesopause region:three-dimensional modeling of their environment and twodimensional modeling of their transport, J. Geophys. Res. Space Phys., 107, SIA 10-1-SIA 10-32, https://doi.org/10.1029/2001JA000316, 2002.

Dalin, P., Kirkwood, S., Hervig, M., Mihalikova, M., Mikhaylova, D., Wolf, I., and Osepian, A.: Wave influence on polar mesosphere summer echoes above Wasa: experimental and model studies, Ann. Geophys., 30, 1143-1157, https://doi.org/10.5194/angeo-30-1143-2012, 2012.

Dillmann, A. and Meier, G. E. A.: A refined droplet approach to the problem of homogeneous nucleation from the vapor phase, J. Chem. Phys., 94, 3872-3884, https://doi.org/10.1063/1.460663, 1991.

Duft, D., Nachbar, M., and Leisner, T.: Unravelling the microphysics of polar mesospheric cloud formation, Atmos. Chem. Phys., 19, 2871-2879, https://doi.org/10.5194/acp-19-2871-2019, 2019.

Gail, H.-P. and Sedlmayr, E.: A\&A, 132, 163, https://doi.org/1984AA...132..163G, 1984.

Gumbel, J. and Merner, J.: Charged meteoric smoke as ice nuclei in the mesosphere: Part 1 -A review of basic concepts, J. of Atmos. and Solar-Terrestrial Phys., 71, 1225-1235, https://doi.org/10.1016/j.jastp.2009.04.012, 2009.

Harvey, A. H. and Lemmon, E. W.: Correlation for the Second Virial Coefficient of Water., J. Phys. Chem. Ref. Data, 33, 369-376, https://doi.org/10.1063/1.1587731, 2004.

Hervig, M. E., Deaver, L. E., Bardeen, C. G., Russell, J. M., Bailey, S. M., and Gordley, L. L.: The content and composition of meteoric smoke in mesospheric ice particles from SOFIE observations, J. Atmos. Sol. Terr. Phys., 84-85, 1-6, https://doi.org/10.1016/j.jastp.2012.04.005, 2012.

Hunten, D. M., Turco, R. P., and Toon, O. B.: Smoke and dust particles of meteoric origin in the mesosphere and stratosphere, J. Atmos. Sci., 37, 1342-1357, https://doi.org/10.1175/1520-0469(1980)037<1342:SADPOM>2.0.CO;2, 1980.

Kalikmanov, V. I.: Nucleation theory, vol. 860 of Lecture Notes in Physics, Springer, Dordrecht, 2013. 
Kouchi, A., Yamamoto, T., Kozasa, T., Kuroda, T., and Greenberg, J. M.: Conditions for condensation and preservation of amorphous ice and crystallinity of astrophysical ices, A\&A, 290, 1009-1018, https://doi.org/hdl.handle.net/2115/42838, 1994.

Laaksonen, A., Ford, I. J., and Kulmala, M.: Revised parametrization of the Dillmann- Meier theory of homogeneous nucleation, Phys. Rev. E, 49, 5517, https://doi.org/10.1103/PhysRevE.49.5517, 1994.

Lübken, F.-J.: Thermal structure of the Arctic summer mesosphere, Geophys. Res. Let., 104, 9135-9149, https://doi.org/10.1029/1999JD900076, 1999.

Lübken, F.-J., U., B., and G., B.: On the Anthropogenic Impact on Long-Term Evolution of Noctilucent Clouds, J. of Geophys. Res, 45, 1-9, https://doi.org/110.1029/2018GL077719, 2018.

Lübken, F.-J. Zecha, M., Hoffner, J., and Rottger, J.: Temperatures, polar mesosphere summer echoes, and noctilucent clouds over Spitsbergen (78 degrees N), Geophys. Res. Let., 109, D11 203, https://doi.org/10.1029/2003JD004247, 2004.

Manka, A., Pathak, H., Tanimura, S., Wölk, J., Strey, R., and Wyslouzil, B. E.: Freezing water in no-man's land, Phys. Chem. Chem. Phys., 14, 4504-4516, https://doi.org/10.1039/c2cp23116f, 2012.

Megner, L., Rapp, M., and Gumbel, J.: Distribution of meteoric smoke - sensitivity to microphysical properties and atmospheric conditions, Atmos. Chem. Phys., 6, 4415-4426, https://doi.org/www.atmos-chem-phys.net/6/4415/2006/, 2006.

Megner, L., Siskind, D. E., Rapp, M., and Gumbel, J.: Global and temporal distribution of meteoric smoke: A two-dimensional simulation study, J. Geophy. Res., 113, D03 202, https://doi.org/10.1029/2007JD009054, 2008.

Murphy, D. M. and Koop, T.: Review of the vapour pressures of ice and supercooled water for atmospheric applications, Q. J. R. Meteorol. Soc., 131, 1539-1565, https://doi.org/10.1256/qj.04.94, 2005.

Murray, B. J. and Jensen, E.: Homogeneous nucleation of amorphous solid water particles in the upper mesosphere, J. of Atmos. and SolarTerrestrial Phys., 72, 51-61, https://doi.org/10.1016/j.jastp.2009.10.007, 2010.

Ostwald, W.: Z. Phys. Chem., 22, 289-330, 1879.

Plane, J. M. C., Feng, W., and Dawkins, E. C. M.: The Mesosphere and Metals: Chemistry and Changes, JChem. Rev., 115, 4497-4541, https://doi.org/10.1021/cr500501m, 2015.

Rapp, M. and Lübken, F. J.: Polar mesosphere summer echoes: review of observations and current understanding, Atmos. Chem. Phys., 4, 2601-2633, https://doi.org/10.5194/acp-4-2601-2004, 2004.

Rapp, M. and Thomas, G. E.: Modeling the microphysics of mesospheric ice particles: Assessment of current capabilities and basic sensitivities, J. Atmos. Sol. Terr. Phys., 68, 715-744, https://doi.org/10.1016/j.jastp.2005.10.015, 2006.

Rapp, M., Lübken, F. J., Müllemann, A., Thomas, G. E., and Jensen, E. J.: Small-scale temperature variations in the vicinity of NLC: Experimental and model results, J. Geophys. Res., 107, AAC-11, https://doi.org/doi.org/10.1029/2001JD001241, 2002.

Tanaka, K. K., Tanaka, H., and Nakazawa, K.: Non-equilibrium Condensation in a Primordial Solar Nebula: Formation of Refractory Metal Nuggets, Icarus, 160, 197-207, https://doi.org/10.1006/icar.2002.6960, 2002.

Tanaka, K. K., Kawano, A., and Tanaka, H.: Molecular dynamics simulations of the nucleation of water: Determining the sticking probability and formation energy of a cluster, J. Chem. Phys., 140, 114 302(1-10), https://doi.org/10.1063/1.4867909, 2014.

Vaste, O.: Noctilucent clouds, Journal of Atmospheric and Terrestrial Physics, 55, 133-143, https://doi.org/10.1016/0021-9169(93)90118-I, 1993.

Yamamoto, T. and Hasegawa, H.: Grain formation through nucleation process in astrophysical environment, Prog. Theor. Phys., 58, 816-828, https://doi.org/10.1143/PTP.58.816, 1977. 
https://doi.org/10.5194/acp-2021-728

Preprint. Discussion started: 14 October 2021

(C) Author(s) 2021. CC BY 4.0 License.

(c) (1)

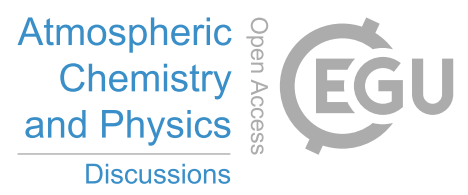

Zasetsky, A. Y., Petelina, S. V., and Svishchev, I. M.: Thermodynamics of homogeneous nucleation of ice particles in the polar summer mesosphere, Atmos. Chem. Phys., 9, 965-971, https://doi.org/10.5194/acp-9-965-2009, 2009. 